\title{
WCSC 2019: the 9th World Chess Software Championship
}

Article

Accepted Version

The ICGA WCSC 2019 report

Krabbenbos, J., van den Herik, J. and Haworth, G. (2020) WCSC 2019: the 9th World Chess Software Championship. ICGA Journal, 41 (4). pp. 222-236. ISSN 1389-6911 doi: https://doi.org/10.3233/ICG-190126 Available at https://centaur.reading.ac.uk/85846/

It is advisable to refer to the publisher's version if you intend to cite from the work. See Guidance on citing.

Published version at: https://content.iospress.com/articles/icga-journal/icg190126

To link to this article DOI: http://dx.doi.org/10.3233/ICG-190126

Publisher: The International Computer Games Association

All outputs in CentAUR are protected by Intellectual Property Rights law, including copyright law. Copyright and IPR is retained by the creators or other copyright holders. Terms and conditions for use of this material are defined in the End User Agreement.

\section{www.reading.ac.uk/centaur}

\section{CentAUR}

Central Archive at the University of Reading

Reading's research outputs online 


\section{WCSC 2019: The $9^{\text {th }}$ World Chess Software Championship}

Jan Krabbenbos, Jaap van den Herik and Guy Haworth ${ }^{1}$

Amersfoort, the Netherlands, Leiden, the Netherlands and Reading, UK

The $9^{\text {th }}$ World Chess Software Championship started on August $11^{\text {th }}, 2019$. The six programs of Table 1 participated in a double round robin tournament of ten rounds. The tournament took place at the Venetian Macau Hotel Resort in Macau, China and was organized by the ICGA. The venue was part of the $28^{\text {th }}$ International Joint Conference on Artificial Intelligence (IJCAI-19) which also acted as the main sponsor. The other important sponsor was Digital Game Technology (DGT) who provided the electronic chessboards. Chessbase donated an extra prize for the first position. The WCSC was held in conjunction with the $25^{\text {th }}$ 'WCCC' World Computer Chess Championship and 2019 'WSCC' World Speed Chess Championship. The tempo was $45^{\prime}+15^{\prime \prime} / \mathrm{m}$. Here, we comment on the highlights of all the games, fully available as usual in our supporting files (Krabbenbos et al., 2019a-2019c).

In the WCSC, all programs play on the same hardware platform. This year, the IJCAI provided Lenovo computers with a 1.1 GHz 64-bit Intel Pentium Silver N5000 CPU, Windows 10, 4 GiB internal memory and a 128 GB SSD - adequate if not able to host all 7-man endgame tables.

Table 1

The participants in WCSC 2019 (CPW, 2019)

\begin{tabular}{ccccc}
\hline id & Program & Author(s) & CC & Operator \\
\hline C & CHIRON & Ubaldo Andrea Farina & IT & Ubaldo Andrea Farina \\
G & GINKGO & Frank Schneider & DE & Wolfgang Zugrav \\
J & JONNY & Johannes Zwanzger & DE & Johannes Zwanzger \\
K & KOMODO & Don Dailey, Larry Kaufman \& Mark Lefler & US & Erdogan Günes \\
S & SHREDDER & Stefan Meyer-Kahlen & DE & Stefan Meyer-Kahlen \\
T & THE BARON & Richard Pijl & NL & Richard Pijl \\
\hline
\end{tabular}

Round 1: GINKGO - THE BARON 1-0, CHIRON - JONNY 1/2-1/2, KOMODO - SHREDDER 1-0.

The game GINKGO - THE BARON followed well-trodden paths. The opening was a Queen's Gambit Accepted. Nowadays the moves are available from a variety of opening books. GINKGO was the first to leave its book but THE BARON continued to move 21 . There was further material available but it was simply not incorporated in either program. The HIARCS book continues a line up to move 27, in fact the game continuation, and even gives another four moves. The evaluation of the position by both sides at move 21 was equal. From move 21 to 30, there was nice interplay of small combinations which did not destroy equality. After White's $30^{\text {th }}$ move, we came to a queen and rook ending: insiders speculated it would end in a draw. However, the unpredictable computers made unexpected decisions which influenced the material balance if not the outcome of the game. From Fig. 1a, Black played $\mathbf{3 0}$. ... Rhd8, inviting White to follow an interesting line. White took up the challenge and played $\mathbf{3 1}$.

\footnotetext{
${ }^{1}$ Corresponding author: g.haworth@ reading.ac.uk
} 
Rg3+ whereafter Black played 31.... Qxg3 32. fxg3 Rxd7. Exact evaluation is difficult but common chess knowledge teaches us that a draw is the foreseeable outcome.

Taking decisions in an unbalanced situation is difficult and the next 20 moves saw White playing dynamically with the queen which resulted in an exchange of many pawns and an endgame with two separated ('a' and ' $\mathrm{g}$ ') pawns and a queen versus two rooks. The strategic line for chess players was straightforward: defend with two rooks on the fifth row with the king behind. Even so, THE BARON was hampered by that the fact that, according to its author Richard Pijl: 'THE BARON believed it was two pawns down.' Henceforth all decisions taken were influenced by this knowledge. As a consequence, the black king became active, crossed the middle line of the chessboard and walked to the corner square h1. At that moment White outplayed the black position by a simple mating threat, see Fig. 1b.

Black's 92. ... Rc3+ is forced and then the white pawns become mobile again and hence a threat. Within ten moves, the a- and c-pawns had progressed one square each. At that time, Black resigned and Wolfgang Zugrav accepted the resignation. For the readers it is interesting to know that Erdogan Günes checked the 7-man tables on his iPhone - a win for White, $d t m / z=38 / 3$ moves.

The game CHIRON - JonNy started very quietly with the Giuoco Piano. Pieces were placed on the right squares and around move 14, JONNY was out of book. It played 14. ... Qe7 and from that moment had to find its own way. The first crucial moment arose after 27. ... Qa3, see Fig. 1c. The normal move would have been 28. Ra1. It was not clear what White had in mind when playing 28. b6. Black won a pawn but it was not sufficient for a win. White defended accurately and in a rook endgame of two pawns versus three, it managed to draw the game.
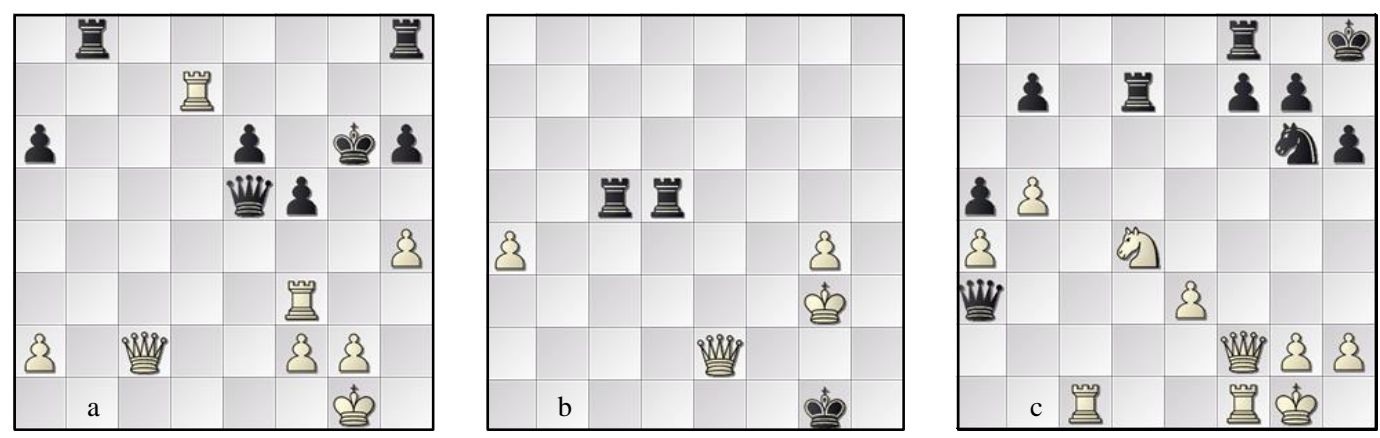

Fig. 1. Round 1: (a) GINKGO - THE BARON position 30b and (b) 92b $(d t m / z=50 / 4 m)$; (c) CHIRON - JONNY $28 \mathrm{w}$.

World champion KOMODO was playing the former world champion SHREDDER. It was a Slav game in which they followed a well-known opening line up to move 13.... a5. The first interesting point in the game appeared on the fifteenth move when Black played 15... 0-0, see Fig. 2a.

It is difficult to see that castling kingside leads Black into trouble. A deeper analysis weighing the pros and cons reveals that White can easily stop the black attack on the queenside but Black has more difficulties in stopping the aggressive manoeuvers by White on the kingside. To profit from the ideas described above requires professional insight. The White program clearly showed its abilities in this respect. On move 23, White achieved its goal by playing 23. e5, see Fig. 2 b.

It still took more than 20 moves before White progressed towards its goal of weakening some black squares and occupying them without any risk of neutralizing exchanges, see Fig. 2c. White took the 
initiative by playing 44. Nc5. After a shuffling of rooks and bishops, White managed to penetrate the black position. With the manoeuver Qb5-a4, it created a passed pawn that was unstoppable. Note that the exchange of queens was forced and that the passed a-pawn was an irresistible asset for White. Black resigned after few more moves.

After R1: 1= GINKGO, KOMODO 1; 3= CHIRON, JONNY 1/2; 5= SHREDDER, THE BARON 0.
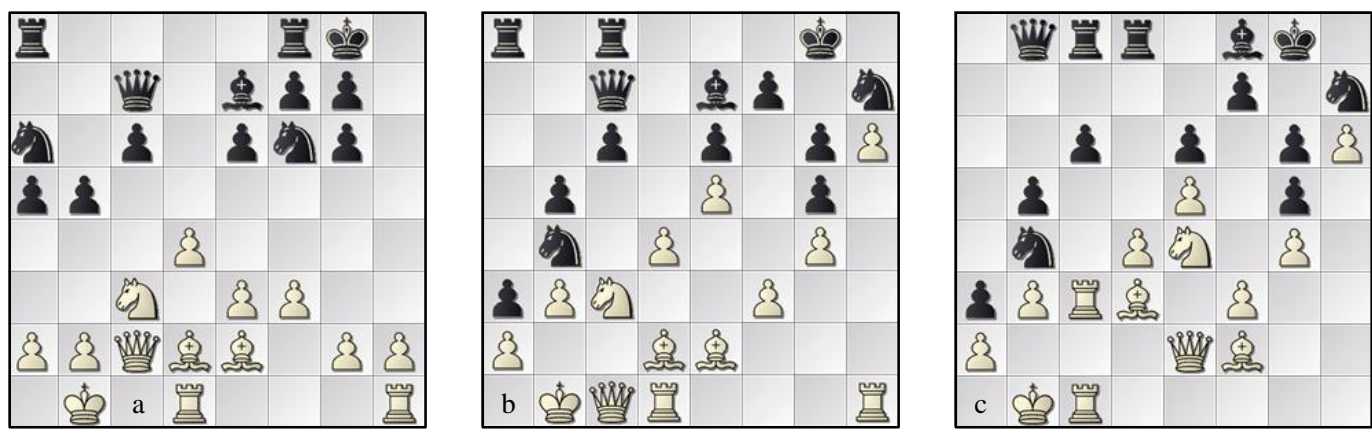

Fig. 2. Round 1: KoMODO - SHREDDER (a) 16w, (b) 23b and (c) 44w.

\section{Round 2: THE BARON - SHREDDER 1/2-1/2, JONNY - KOMODO 1/2-1/2, GINKGO - CHIRON 1/2-1/2.}

THE BARON - SHREDDER followed the same opening as KOMODO - SHREDDER in the first round. However, SHREDDER had learned its lessons and did not consider kingside castling this time. Therefore the game developed along completely different lines. After a series of exchanges, the strategic threat, which had typified SHREDDER's game in the first round, had disappeared and castling kingside was possible, see Fig. 3a.

Tactical issues are not a characteristic of this position and soon the game found itself in a quiet place. After 45. Rd1, an implicit exchange of rooks took place. The ensuing endgame was drawn but its realization took some time. It almost started to be exciting when Black managed to force the white king to march to Black's back rank. Black's unsupported queen was no danger to the white king and the endgame complications did not threaten either side.

JONNY - KOMODO did not excite the public though it should be admitted that opponents of less than grandmaster strength would be impressed by the manoeuvers of either side. Deeper insight into the position clearly shows that the balance never showed the slightest excursion from equilibrium. After 50 moves, the opponents agreed to a draw.

GINKGO - CHIRON started as Giuoco Piano, one of the favourite openings of this championship. Up to move 32, there was no excitement to report in this game. Black had obtained some small opportunities and believed with its 32. ... Bh3 that it was time for action as in Fig. $3 \mathrm{~b}$.

After 33. Re1 g4 34. Qd2, Black played the interesting move 34.... g5. This manoeuver gave White the opportunity to unbalance the pawn structure. It resulted in Black having a locked bishop and a passed pawn and White having a breakthrough possibility on the queenside. With ingenious play, White managed to transform its breakthrough with a passed pawn on the queenside. However, closer analysis showed that the locked bishop was still a powerful piece and so White decided to exchange bishops. After pushing the passed a-pawn to the seventh rank, the position became double-edged. White must have seen its defensive possibilities some time in advance so the move 54. h4 as in Fig. $3 \mathrm{c}$ did not come as a surprise for either side. 
White had computed very well that the two black passed pawns did not entail a real threat since the rook sacrifice on b6 was an essential part of its plan. Hence, Black played 54. ... Rhe8. After some manoeuvring, White managed to give perpetual check and reach the draw.

After R2: 1= GinkGO, KOMODO 11/2; 3= CHIRON, JONNY 1; SHREDDER, THE BARON 1/2.
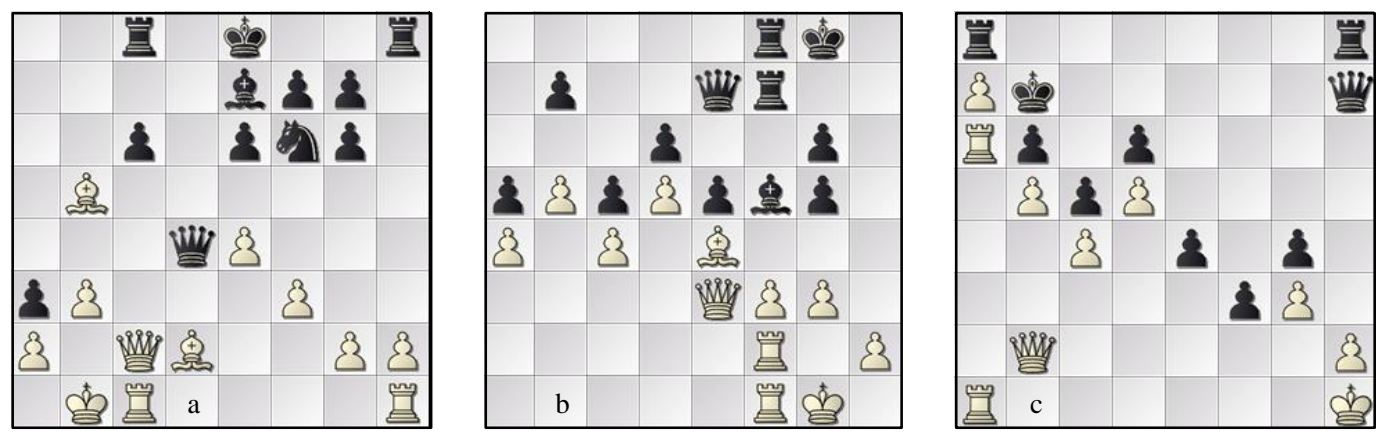

Fig. 3. Round 2: (a) THE BAron - Shredder 21b; (b) GinKGO - Chiron 32b and (c) 54w.

\section{Round 3: CHIRON - THE BARON 1-0, KOMODO - GINKGO 1/2-1/2, SHREDDER - JONNY 1/2-1/2.}

The game CHIRON - THE BARON was a Catalan variation in which THE BARON optimistically played 10. ... f5, see Fig. 4a. At the time of the play, the disadvantages were not clear. However, it soon transpired that White could develop its pieces quite easily and exchange the right pawns in the center. It all resulted in a weak pawn on e6. The piece activity of Black was in the first instance acceptable compensation but after annihilation by White, the weak pawn on e6 attracted many white pieces as in Fig. 4b.

In the end, the pawn was lost with 29. Qxe6, no compensation was to be found and White regrouped the pieces for a final attack on the queenside in combination with a progressing passed pawn. This lead to resignation by THE BARON.
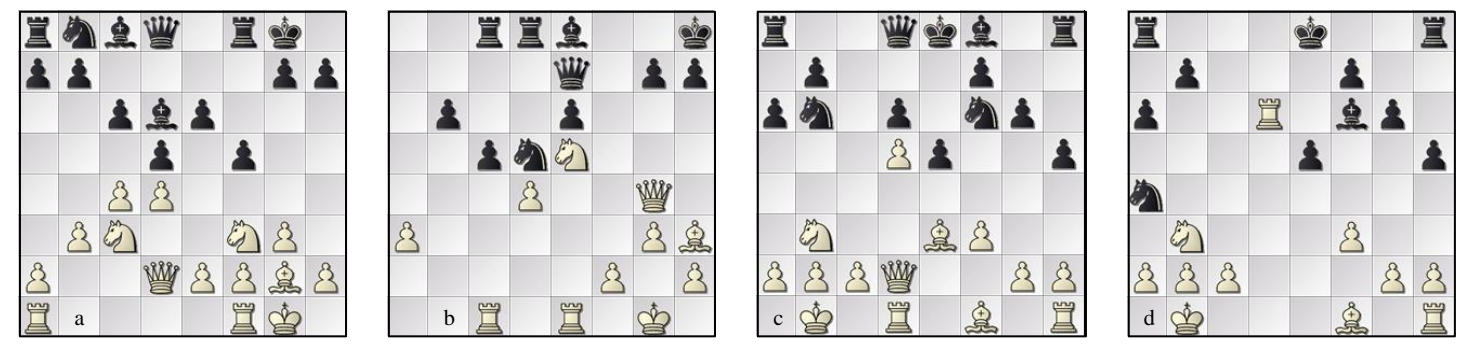

Fig. 4. Round 3: (a) CHIRON - THE BARON 11w and (b) 29w; (c) KoMODO - GinKGO 13b and (d) 19w.

The game KomODO - GINKGO was a Sicilian game directly in line with modern fashion with the choice of taking the pawn on $\mathrm{d} 5$ as in Fig. 4c. Wolfgang Zugrav stated that the usual move Nbxd5 leads to interesting games and the other move Nfxd5 to an equal endgame. There was no incentive given to the program but it chose the simple game. It relied on a small trick which occurred after $\mathbf{1 8 .}$ ... Na4, see Fig. 4d. The move 19. Rxf6 is impossible because of 19. ... Ke7. The situation implied a fast regrouping of the pieces and an exchange of rooks and pawns. The resulting endgame of oppositecolour bishops with equal numbers of pawns was a clear draw. 
The game SHREDDER against JONNY was one of many encounters, the exact number not in fact known to the author/operators Stefan Meyer-Kahlen and Johannes Zwanzger. In the Slav opening, Black relied on fast progress for the queenside pawns. The move 13. ... c5 was not expected by SHREDDER, see Fig. 5a. The continuation was 14. dxc5 Rc8 15. axb4 axb4 16. Bxh7+ Nxh7 17. Rxd6 - a surprising series of moves for human beings but the computer programs computed this line as equal. Still, the configuration was characterized by unprotected rooks and pieces as in Fig. 5b.

White played 20. Nb6. The game continued 20. ... Rc7 21. Ra5 Qe5 22. Rd2 b3 23. Qc4 Rb8 24. Rb5 Qf5 and the dance of the pieces found its pinnacle with 25. Nd5, see Fig. 5c. It is remarkable that neither program was impressed by their opponent's threats but continued with their own plans. This was the best way and led to an equal position. The queens came off and the triple pawn of White was not in fact a disadvantage. Twenty more moves saw the draw agreed.

After R3: 1= CHIRON, GINKGO, KOMODO 2; 4 JONNY 11⁄2; 5 SHREDDER 1; 6 THE BARON 1².
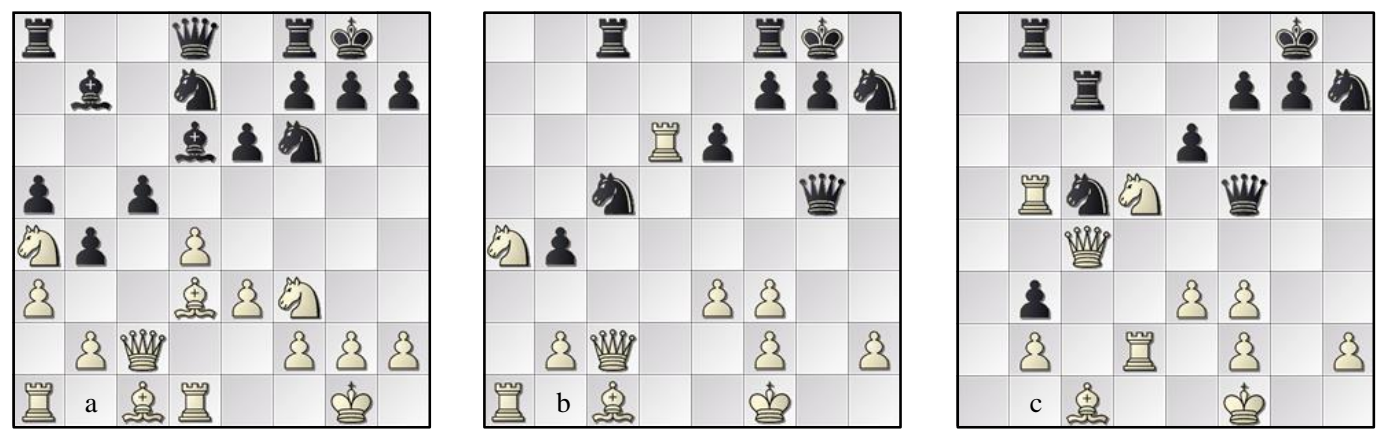

Fig. 5. Round 3: SHREDDER - JONNY (a) 14w, (b) 20w and (c) $25 \mathrm{~b}$.

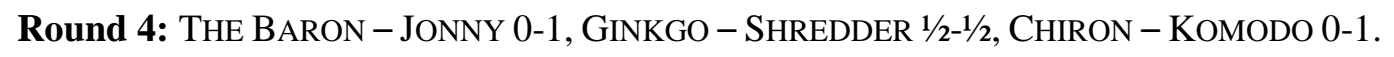

THE BARON - JONNY showed that opening playing style and strategic decisions should be in harmony during the preparation of the game. Every move is a decision and five decisions in a row cannot be played without harmony. In the beginning, it is not clear why the moves are not in harmony, but an experienced program is tuned to reveal any weaknesses deriving from the incompatibility of strategy and structure. In the current game, the move 11. f4, see Fig. 6a, looks logical but it turned out to be a weakness in the later middlegame. This was clearly exploited by Black by the following strategy: (a) exchanging the $\mathrm{f} 4$ pawn so that, after 25. exf4, the new white f4 pawn would need support from the g-pawn and then (b) use squares e4 and g4 for its knight. This was easily expressed by the commentator and in this case was also straightforwardly exploited by JONNY.

The approach chosen by Black finally resulted in a position where $\mathbf{2 4}$. $\mathbf{g 3}$ had to be played, see Fig. 6b. The continuation was 24. ... gxf4 25. exf4 e5 26. Qf2 Qa5 27. Nc3 Ng4 28. Qe2 Qc5+. At this point, Black had full control of the important squares and penetrated the white position within five moves. Eventually, White resigned.

The game GINKGO - SHREDDER started with a Sicilian opening and then followed the well-known Scheveningen variation. The dynamics of the game require utmost alertness from both sides: a small step away from the line of equilibrium can be fatal. For both sides the intriguing question in the context of Fig. 6c was "What is the value of 41. Bd6?" 
Obviously Black had to sacrifice the exchange for a pawn and both programs assumed that this all happened in the draw zone. As the game continued, it looked like that this had been the case. We advise the reader to replay the game on the board so that you will arrive at the final position in which a draw was agreed, Fig. 6 d.

The endgame tables of both programs agreed on the draw result so this was the official outcome. Closer inspection revealed that the current position was both 'mate in 79 moves' and a 50-move-rule draw: $d t z=65 \mathrm{~m}$. This raises the question of how current computer programs handle the endgame tables. In the case at hand, SHREDDER had the parameters set to observe the 50-move rule. All in all a nice game, an interesting result but in general, one would not be wise to follow this line as a slight misstep can have huge consequences for the outcome.
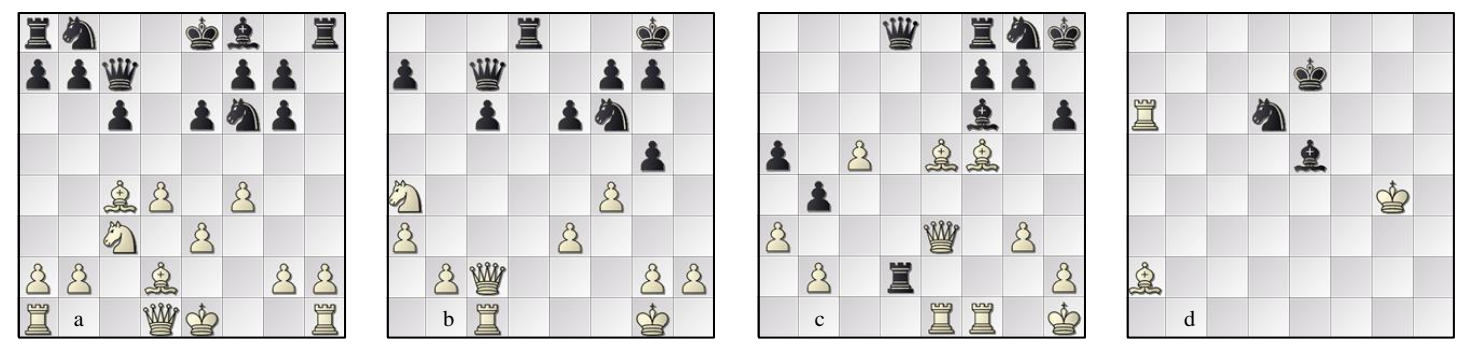

Fig. 6. Round 4: (a) THE BARON - JONNY 11b and (b) 24w; (c) GiNKGO - SHREDDER 41w and (d) 74b.

CHIRON - KOMODO was a fantastic game. It was a spectacular fight for the initiative in which CHIRON was active on the kingside and KOMODO on the queenside. The activity after the opening showed a great deal of subtlety. White emphasized a kingside attack and did not care about the loss of a pawn on the queenside, see Fig. 7a. Black was convinced that its kingside pieces were well positioned for defending against White's threats. Therefore, it took time to take the white pawn on a6. White targetted its own goal with the line 28. 55 Nh7 29. f4 Rb6 30. g4 a5 31. f5 while Black was active on the queenside and pushed its pawns forward energetically. But who would come out on top?

White saw a nice combination and played 36. e6, Fig. 7b, followed by 36. ... fxe6 37. Rxc4. The board was on fire with ideas everywhere for successful continuations. Andrea thought CHIRON missed the draw, starting with 40. Rc1? Here Black replied 40.... b3, Fig. 7c, increasing its queenside attack and promotion threat. The position became more excited after 41. Qxc6 Rb8 42. Bg3 'not optimal' (Andrea) Qd8 43. d5 Rb6 44. Qc4 'the real blunder' (Andrea) b2, Fig. 7d.
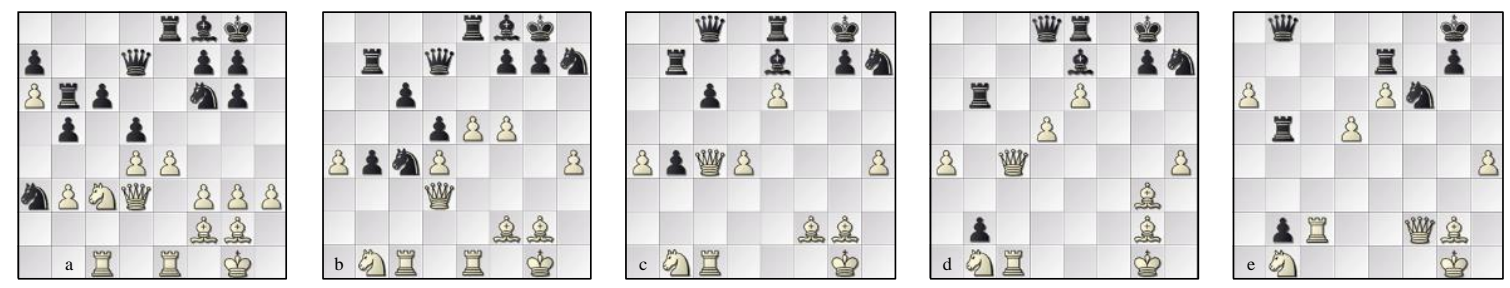

Fig. 7. Round 4: CHIRON - Komodo (a) 27b, (b) 36w, (c) 40b, (d) 45w and (e) 51w.

Time for reconsideration and repositioning of the pieces on both sides. White used its a-pawn asset: 45. Rc2 Bd6 46. Bxd6 Qxd6 47. Qc5 Qb8 48. a5 Rb5 49. Qf2 Nf6 50. a6 Re7, Fig. 7e. The last move definitely stopped the advancement of the a-pawn. White had to take into the account the 
possibilities of the black knight and did not find a good solution. The game continued $\mathbf{5 1 .}$ Kh1 Ng4 52. Qg1 Qf4 53. Bh3 Qe4+ 54 Qg2 Nf2+ 55. Rxf2 Qxb1+ and White resigned.

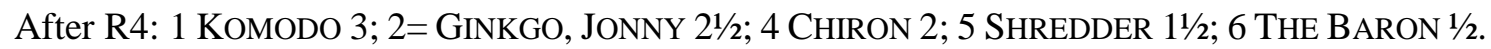

Round 5: KOMODO - THE BARON 1-0, SHREDDER - CHIRON 1/2-1/2, JONNY - GINKGO 1/2-1/2.

KOMODO - THE BARON was a game in which THE BARON showed its best side: playing, playing and playing. It is a game which we can nowadays replay very quickly on a computer and sense the feeling Black must have had when it became aware that its defense would not be sufficient for a draw. The game required 134 moves and reached a QP(f)-Q endgame on move 65. THE BARON sometimes received its endgame table information incorrectly and occasionally thought the game a draw. The reality was otherwise: KOMODO won.

SHREDDER - CHIRON played a semi Tarrasch with a fast exchange of queens. The knights were very active either to defend or to regain a pawn. After the opening fight was over the game turned to calmer waters in which both sides found themselves well. There were no exciting moments and good technique from both ensured that the deserved draw was never far away.

JONNY - GINKGO was a Giuoco Piano with adequate play from both sides. How piano the game was is not clear as Black managed to achieve a position a pawn up. At the beginning, White had some counterplay but that disappeared after the exchange of many pieces, rooks and pawns. Finally, the game arrived at a rook ending with three pawns versus two. It required precise defense but there was never a possibility to obtain more than a draw - the result after 62 moves.

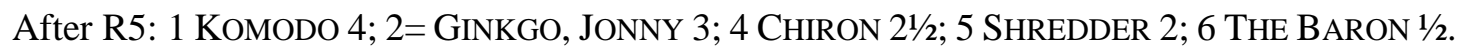

Round 6: THE BARON - GINKGO 0-1, JONNY - CHIRON 1/2-1/2, SHREDDER - KOMODO 1/2-1/2.

In THE BARON - GINKGO, Black played the opening somewhat unusually to get THE BARON playing on the basis of only its own ideas. The implicit consideration was of course to seduce THE BARON into playing moves that did not adequately fit the position. To some extent, Black managed to get some positional advantage on the kingside which White hoped to combat with activities on the queenside. However, White could not find the right plan and in Fig. 8a after 25. Bd2, it was clear that the threats as exhibited by Black were more promising than the white ones.

The plan for Black is quite easy, namely bringing all pieces over to the kingside. Whereas for White it is more difficult to move over to the queenside since the entries for penetration are not clear. In the game, the white pieces manoeuvred back and forth. This gave Black the opportunity to perform the final knocking on the white king's door which led to an advantageous end to the game, see Fig. 8b. The game continued with 32.... g3 33. fxg3 hxg3 34. hxg3 Bh8 35. Nb5 Rh7 36. gxf4 exf4 37. Rxd7 Qh2+ and after fifteen more moves, White resigned.

In the game JONNY - CHIRON the English opening led to an even position at the beginning of the middlegame. Yet both programs were rather ambitious, searching for possibilities to deploy some action. White acted cautiously but Black showed more power in its manoeuvres. It resulted in White being on the defensive as in Fig. 8c; care was of the utmost importance.

White must reply to the move 31.... Bxg4. It did not play the obvious 32. Nxg4 but 32. e3. That was a clever defense. The game continued 32. ... Bh3 33. exd4 Bxd4 34. Kh1 Bxb2 35. Qxb2 Ra2 36. Qd4 Qxf2 37. Qd8+ Qf8 38. Qd5+ Qf7 39. Qxf7 Kxf7 and entered into a drawn endgame. 
SHREDDER - KOMODO was a contest between two strong players, neither of which would yield easily. They fought for power in the center and looked for small advantages. However, their play was also directed at annihilating potential threats so the game continued without real excitement. Their several clever procedures led to a difficult endgame with a rook each plus bishop versus knight. Here also, the contenders stood toe to toe in equal contest until the draw was agreed.

After R6: 1 KoMOdO 41/2; 2 GINKGO 4; 3 JONNY 31/2; 4 CHIRON 3; 5 SHREDDER 21/2; 6 THE BARON 1².
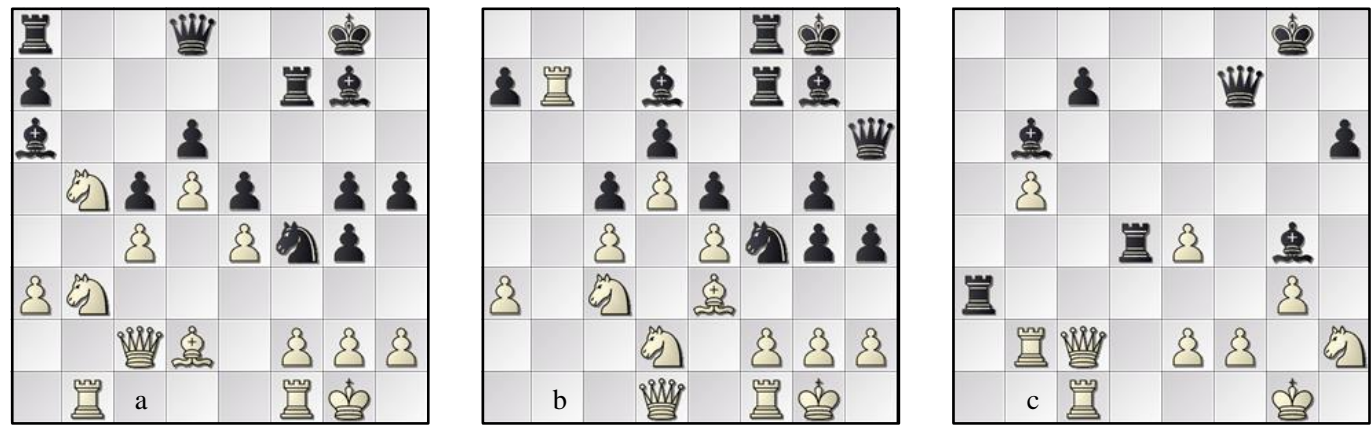

Fig. 8. Round 6: (a) THE BARON - GINKGO 25b and (b) 32b; (c) JONNY - CHIRON 32w.

\section{Round 7: SHREDDER - THE BARON 1-0, KOMODO - JONNY 1/2-1/2, CHIRON - GINKGO 1/2-1/2.}

The game KOMODO - JONNY was an important meeting for the first place. Operator Erdogan expressed the opinion that playing a standard opening usually transitions to an equal middlegame. Trying to win means taking calculated risks. This game is an example of such a strategy. It almost worked well but JONNY was able to hold its own and so could constrain the white advantage during the game to no more than 0.65 in terms of the evaluation function. The first deviation from the main line of the book opening, that is, by choice of sideline, was 8. g3. Thereafter both players positioned their pieces on relevant squares without deep thoughts or strategic lines in mind. Erdogan: 'The first idea was 16. $\mathrm{Ne} 1$ with as strategic follow up f2-f4-f5 and having the opportunity of Qh5. As we know, JONNY is also a strong program and took adequate countermeasures against this line, see Fig. 9a and its continuation.

To prevent White from the strategic line mentioned above, Black becomes active successfully on the queenside as we will see: 16. ... Ra8 17. Rxa8 Qxa8 18. Nc2. Here, 18. f4 was to be considered. By playing 21. Qf3, White showed that it was prepared to change plans although it maintained the idea that the king attack should be the key to a victory. A tough fight followed, see Fig. 9b.

The continuation was 24. ... exf4 25. Bc3+ f6 26. Qd2 Qb3. Now the question is how does White follow up its aggressive ideas. KOMODO's analysis showed a preference for a regrouping of the pieces with a clear eye on the black king. 27. Bf3 Ra8 28. Bd1 Qa2 29. Bb2 c5 30. h4 h6 31. bxc5 bxc5 32. Bg4 Qb3 and we are at Fig. 9c.

After this regrouping White calculated the time to be right for a new aggressive action and played 33 . gxf4. Black followed with 33. ... Ra2 34. Rf2 Nxf4 35. Bxd7 taking us to Fig. 9d.

For humans this position might be difficult to analyze, for computers the evaluation is achieved by direct lines which show that White is still $\sim 0.5$ pawn better. Yet White most proceed along a narrow path. 35. ... Kf7 (the threat was Bxf6+) 36. Bf5 Qxd3 37. Kh2 Ke8 38. Bg4 Kf7 39. Bd7 Qb3 40. hxg5 hxg5 41. Bf5 Qd3 42. Qc2 g4 43. Bxg4 Qxc2 44. Rxc2 Nd3. This looks like a difficult situation 
for White but deeper analysis shows that it is Black who has to fight for a draw. Black did so successfully. The draw implied that KOMODO remained the leader of the pack.
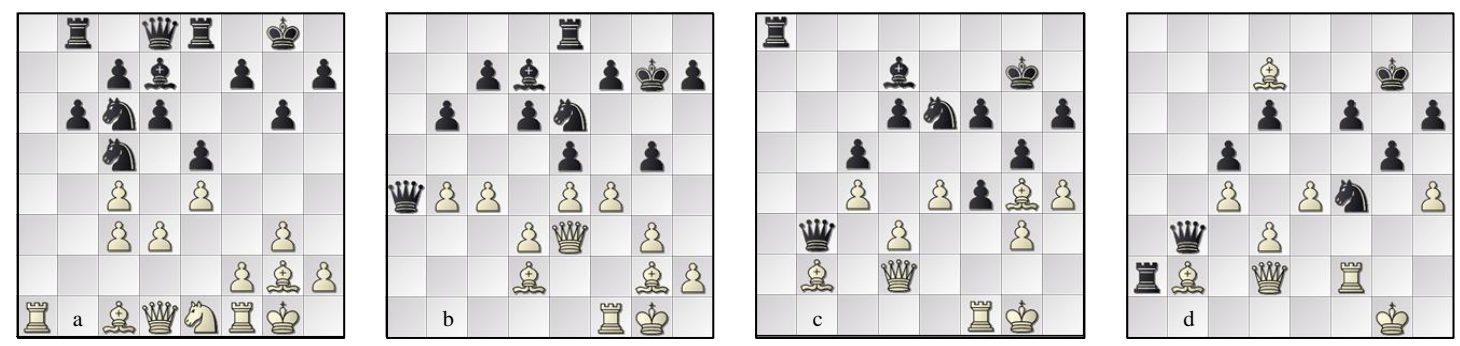

Fig. 9. Round 7: KOMODO - JONNY (a) 16b, (b) 24b, (c) 33w and (d) $35 b$.

CHIRON - GINKGO started with smooth development on both sides. Nowadays it is difficult to leave the opening book with an advantage. Both opening books are too well tuned. The main issue is the strategic handling of the resulting position. Tactically both programs know that there is no gain to be expected. GINKGO was trying to build up a position that promised possibilities for attacks. It took a long time but on move 35 it believed its opportunity was there, see Fig. 10a.

GINKGO decided to sacrifice the exchange for two pawns. The resulting dynamic position was tense but both programs remained calm and White in particular found interesting ways to escape from the attack by Black. Let us have a look at the situation. First, we saw 35. ... Rxb5 36. Bxb5 Rxc2 37. Qxc2 Qxd4+ 38. Qf2 Qc3 39. Re1 Qxa3 40. Qe3 Qb4 41. Bd3 g6 42. e5 Nd5 43. Qf2 dxe5 44. Rxe5 Nf4. After some more moves, Black reached the position of Fig. 10b. The threat is clear and the defense was computer-like.

GINKGO played a normal move 50. ... Qf3 defending the knight on f6. Obviously, gxf3 is not an option nor is Qxg5 because of Qxc6. The game continued as follows: 51. Be4 Qf1 52. Bd5 a3 53. Qd4 h5 54. Bc4 Qf5 55. Rc7 Qa5 56. Ra7 Qb4 57. Ra8 a2 58. Qd3 Nge4. After a second regrouping, it is still clear that Black is looking for an attack in which it could combine its mating threats with queen and knight around the white king (using the other knight for the defence). Generally, and particularly here, a queen and two knights are more dangerous than a queen, a rook and a bishop which do not cooperate as in figure 10c.

65. ... g4 is the start of the last big contest in this game. By the way, it is fair to say that White defended excellently: 66. Be2 Nc3 67. Rxc3 Qd4+ 68. Kh2 Qxc3. This is the best that Black could achieve in this game. GINGKO is a pawn up but it provides nothing. The game lasted another 10 moves before the opponents agreed to a draw.

SHREDDER - THE BARON was a contest between a formal world champion and a former runner up. Although the heydays of SHREDDER are perhaps in the past, it still can play convincingly especially when its opponent offers all kinds of possibilities. THE BARON played sharply with the black pieces. This was just what SHREDDER hoped for. In a simultaneous exchange of pieces, Black was led to a lost position. In the situation of Fig. 10d, we see the run up to the winning combination.

By playing 24. Bd5 White fixed the bishop on $\mathrm{d} 7$ indefinitely. This can best be seen from the game line. Black played 24. ... Qxh4 25. Bf3 b4 26. Rc5 (to prevent Bb5) Qh1+ 27. Ke2 0-0. This is the only move but it loses a piece and thus the game. 
After R7: 1 Komodo 5; 2 GINKGO 41/2; 3 JONNY 4; 4= CHIRON, SHREDDER 31/2; 6 THE BARON 1/2.
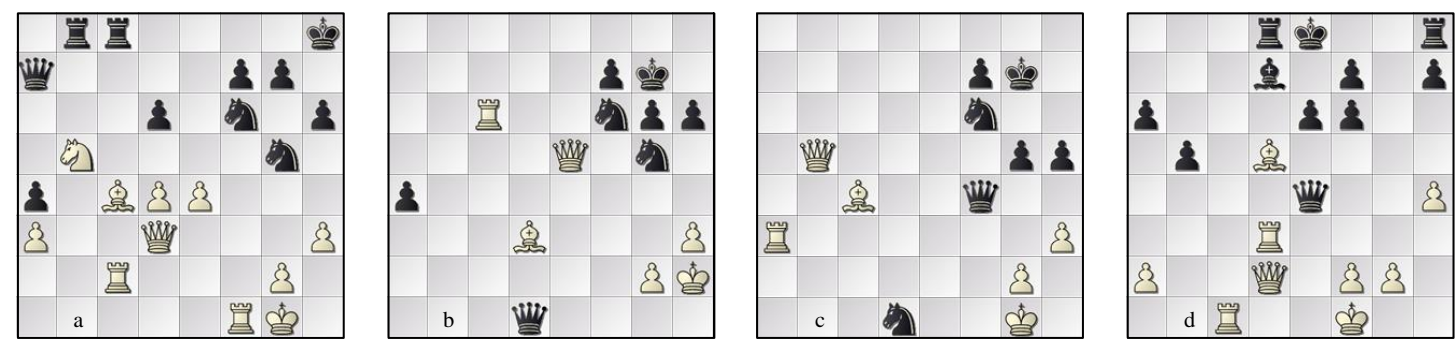

Fig. 10. Round 7: (a) CHIRON - Ginkgo 35b, (b) 50b and (c) 65b; (d) SHREDder - THE BARON 24b.

\section{Round 8: THE BARON - CHIRON 0-1, GINKGO - KOMODO 1/2-1/2, JONNY - SHREDDER 1/2-1/2.}

THE BARON - CHIRON was a clear demonstration of the difference in playing strength. THE BARON played White, had a good position after the opening and then had to find its own way. Creativity should still be guided by the best AI there is otherwise you can see what will happen to your plans. It was a simple misestimation of the disadvantages of putting the black queenside pawns on the black squares. The main task Black was given was the exchange of the black-squared bishops so that the pawns missed their defender. That was strategically the main issue. A rearrangement of the black pieces was necessary but also easy achieved. This implied that the win was easy in this case.

Here, we focus on two positions. Fig. 11a is the beginning of the mismanagement of the game (putting the white queenside pawns on the black squares). 24. ... Kg8 25. Ng3 g6 26. Ne4 h5 27. Nc5 Bd6 28. Rad1 Bf4, Fig. 11b shows the black pieces about to penetrate the white position: 39. ... f3 40. Kg1 Qe2 and after few more moves, THE BARON resigned.

The game GINKGO - KOMODO was definitely not a grandmaster draw since it lasted 59 moves. However, the fight was not a tough one and further, it was not exciting. Clearly, it was a matter of implicit respect for each other's playing strengths. Along the open e-file, the rooks were exchanged and other pieces disappeared in harmony from the battlefield. The bishop ending was a draw from the beginning up to the variant when the bishops disappeared. The resulting pawn endgame was also a draw. Both players might have been happy with this outcome although it did maintain the lead of the half-point that KOMODO held.

The game JONNY - SHREDDER had a quiet opening. Apparently, both players saved their powers for turbulence that they had in mind. In this phase, SHREDDER played a bit cautiously. Zwanzger informed the commentators about two of the more aggressive moves which were possible. The first one was by SHREDDER: it played 13.... Ng6 in the position of Fig. 11c instead of 13. ... Nf5. The consequences were not severe and it required subtle manoeuvring to exploit the defensive moves. An instance of such an exploitation could, according to Zwanzger, have been 23. b4 in Fig. 11d.

Instead of that move, White played 23. Bd2 and gave Black the opportunity to play 23 .... Rc5 attacking the e-pawn. Thereafter Black turned out to have quite some play: 24. f4 Qg4 25. Qf3 Bf5 26. Be4 Qxf3 27. Bxf3 Rc2 28. Rad1 Rxb2 29. Bc1 Rb3 30. Rxd4 Ne6. It resulted in a situation which was difficult to handle for human beings but not for computers because, despite the unbalanced material, the game was essentially drawn. Fig. 11e after 33. Bxb7 might provoke some ideas on possibilities in unbalanced positions. 
33. ... Rxc1 34. Rxc1 Ne2 35. Kh2 Nxc1 36. Bd5 Re3 37. Bxf7+ Kf8 38. Bg6 Bxg6 39. hxg6 Ne2 40. a4 Nxf4 41. Rf7+ Ke8 42. Rxf4 Rxe5 and after few more moves, a draw was agreed.

After R8: 1 KOMODO 51/2; 2 GINKGO 5; 3= CHIRON, JONNY 41/2; 5 SHREDDER 4; 6 THE BARON 1⁄2.
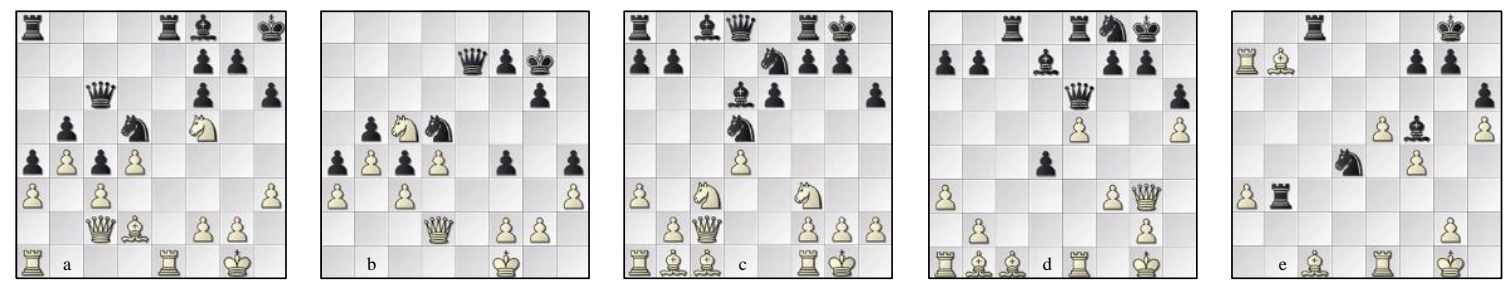

Fig. 11. Round 8: (a) THE BARON - CHIRON 24b and (b) 39b; (c) JONNY - SHREDDER 13b, (d) 23w and (e) 33b.

Round 9: JONNY - THE BARON 1/2-1/2, SHREDDER - GINKGO 1/2-1/2, KOMODO - CHIRON 1/2-1/2.

The leading program KOMODO wanted to show that it really was the best program in the tournament. Therefore, it played actively and energetically against CHIRON. Despite all the trials, CHIRON held its own and was never in peril. KOMODO tried to catch CHIRON unawares by continuations which had already been shown to be harmless. To give you a flavour, we present the position after the opening in which KOMODO has conceived a clearly mighty position in the center of the board. Nevertheless, as stated above, the defensive lines as created by CHIRON were more than sufficient to keep the position equal. After 83 moves, a draw was agreed.

From Fig. 12a, White had continued with 17. d5 which was followed by 17. ... Qe7 18. Rxf4 Bc7 19. Rf1. Sometimes the power in the center is a basis for further progress but in this game, Black managed to annihilate every attempt at such a development. A deserved draw for Black.

JONNY had the idea that THE BARON would help it make a step forward this round. However, THE BARON believed it should continue to go its own way. The well-known English opening by JONNY was met with a pleasant and forceful-looking counter action by Black. Already in the first stage, THE BARON was active on the kingside which was enabled by the early castling to the kingside by White. Professionally JONNY discredited the ideas by THE BARON before they became a threat. On move 19 there was an indirect exchange of bishops which resulted in an almost negligible black pawn on g2 as in Fig. $12 \mathrm{~b}$.
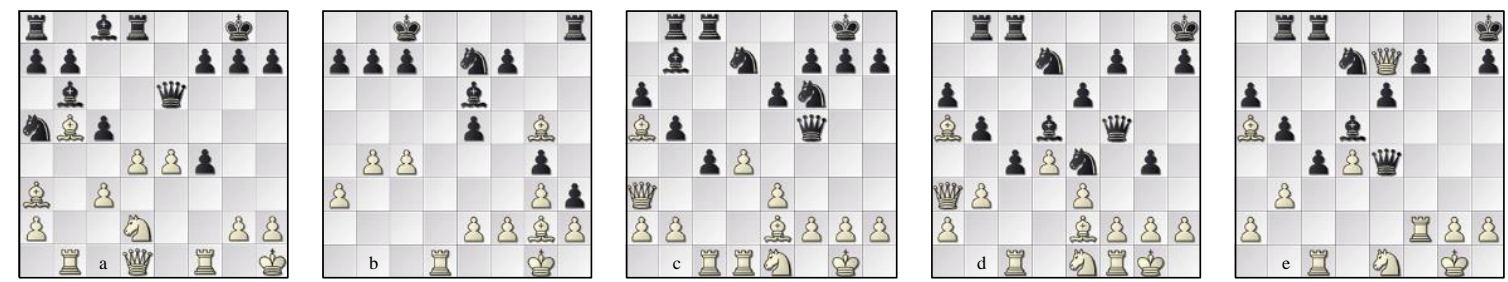

Fig. 12. Round 9: (a) KOMODO - CHIRON 17w; (b) JONNY - THE BARON 19w;

(c) SHREDDER - GiNKGO 20b, (d) 25w and (e) $29 \mathrm{~b}$.

White played 19. Bxe7 followed by 19. ... hxg2 20. Bf6 Re8 21. c5 Bd7 22. f3 e4 23. fxg4 Bxg4 24 . Rd2 e3 25. Rc2 Re6 26. Bg5 f6 27. Bh6 Bf5 28. Re4 Be4 29. h4 Bc6 and now the weak pawn on g2 has changed into a sharp thorn in White's position. This pawn guaranteed the draw whatever White 
tried in the beginning: later, it was Black who played for a win. Even so, it was not sufficient to turn the tables for a win for Black. A deserved half point for THE BARON was the result.

SHREDDER - GINKGO was an exciting game. In brief, the game started with a quiet opening in which both sides tried to seize the initiative. When forces turned out to be rather equal, Black believed aggressive play on the kingside could set the odds in its favour. SHREDDER looked at this development with a positional smile and believed that instead, the advantage should come to it. However, both sides insisted on their strategies and that made the game very interesting. There are many points to highlight in this game. We start with Fig. 12c and the first surprising decision:

Here, Black played 20.... g5. The game continued 21. Nf3 Ne4 22. Rf1 g4 23. Ne1 Kh8 24. b3 Bd5. It looked like Black had a more powerful attack and was now ready to bring over the remaining pieces to the kingside. However, White started to counterattack and follow its own plan for weakening the storm around its king. The first step was $\mathbf{2 5}$. Bxg4 played from Fig. 12d. It was followed by $\mathbf{2 5}$.... Qxg4 26. f3 Qg5 27. fxe4 Qxe3+ 28. Rf2 Qxe4. And now, the finale to this battle: 29. Qe7, Fig. 12c, 29. ... Rb7 30. Bd8 with a threat of Qe8+, Bf6+ and Qxc8. To anticipate this threat, Black replied with a very fine riposte namely 30.... e5 and we are at Fig. 13a.

White can now continue with executing the threat mentioned above but then Black will have two pawns on c4 and $\mathrm{d} 4$ as compensation for the exchange. Therefore, the game continued 31. Qe8+ Kg7 32. dxe5. Here Black replied with 32... Be6. In the new phase of this game, White decided to bring more pieces into the battle around the black king and played 33. Nf3 and the continuation was 33. ... h6 34. Rd1 Nxe5 leading to Fig. 13b.

In this position, White defended itself by 35 . Nxe5 Qxe5 36. Rf3. Black ignored the threat and played 36. ... cxb3 which was naturally followed by $37 . \mathbf{R g 3}+$ and the beautiful 37... Qxg3, see Fig. 13c. After 38. hxg3 bxa2, it turned out that the pawn on a 2 compensated for the difference between a queen and a rook, the material advantage for White.

White continued with 39. Rf1 whereafter Black had another fantastic move, namely 39. ... Rbc7. The game continued with 40. g4 Rc1 41. Bf6+ Kg6 42. Qe7 Rxf1 43. Kxf1 a1Q+ 44. Bxa1 Rc1+ 45. Kf2 Rxa1, the end of the combination, see Fig. 13e.
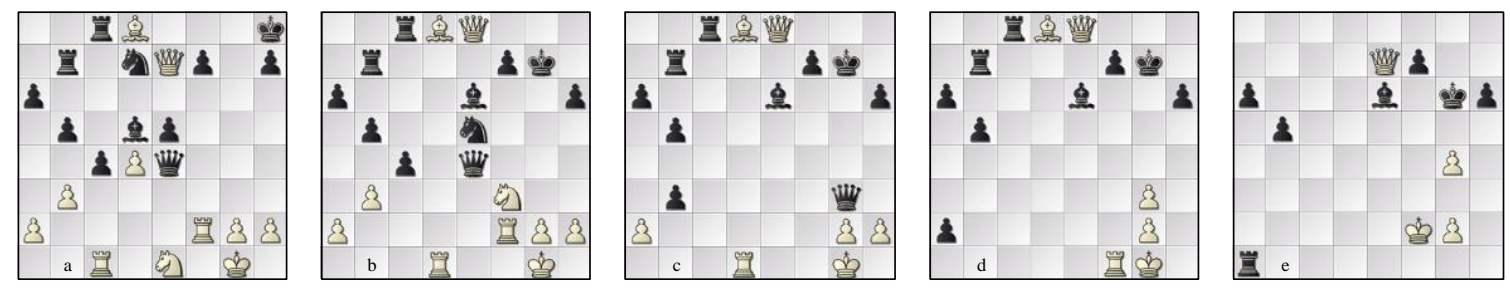

Fig. 13. Round 9: SHREDDER - GiNKGO (a) 31w, (b) 35w, (c) 38w, (d) $39 \mathrm{~b}$ and (e) 46w.

The main question now is 'who is better?' Every experienced chess player may have preferences for the black pieces but will also agree that it is very difficult to achieve advantage in this position. The next 30 moves are a game in itself. Black tries to win by exploiting the passed pawns on the queenside but that is not easy. We here produce the start of the drawing line: 46. Qe8 Ra2+ 47. Kf3 Ra3+ 48. Kf2 Kh7 49. Qe7 Ra4 50. g5 Rf4+ 51. Ke1 b4 52. Qd8 hxg5 53. Qxg5 Rg4 54. Qh5+ Kg7. Black now has a stable position but it is not sufficient for a win so the game continued up to move 74 . At that time, a draw was agreed. 
After R9: 1 KOMOdO 6; 2 GINKGO 51/2; 3= CHIRON, JONNY 5; 5 SHREDDER 41⁄2; 6 THE BARON 1.

Round 10: THE BARON - KOMODO 1/2-1/2, CHIRON - SHREDDER 1-0, GINKGO - JONNY 0-1.

The prize for the most remarkable performance of the tournament goes to THE BARON. It started with 0.5 out of 8 games and then scored one from two with two draws against two of the world's top programs. In the last round, THE BARON faced the current and prospective world champion KOMODO and managed to give the tournament tension to the end. The situation was that KOMODO was leading, just half a point ahead of GINKGO: a draw against THE BARON would give GINKGO the opportunity to draw level on points. THE BARON played a quiet opening and KOMODO attempted to lure it to new territories of play. However, THE BARON played with determination and accepted the pawn sacrifice by Black. That was a correct decision. The counterplay by Black was not much, see Fig. 14a.

White won the pawn by taking on f6. In exchange, Black managed to get control on $\mathrm{d} 4$ because of the following sequence of moves: 18. ... Ne5 19. Bxg7 Rxf1+ 20. Rxf1 Kxg7 21. Nxc5 dxc5 22. Nf3 Nxf3+ 23. Rxf3 Qe5 24. Qc3 Qd4, Fig. 14b.

Here White decided to exchange the queens resulting in an endgame with a pawn up. Of course, the extra pawn is difficult to convert into a winning position and the draw should be reachable. That turned out to be the case. We refer to the moves as played in the game. The result of this outcome is that KOMODO ended on 6.5 out of 10 and could have to tie for first place with GINKGO provided that GINKGO won its game against JONNY. All was still to play for!

The opening between GINKGO and JONNY was quite irregular. GINKGO opened with 1. c4 and JONNY answered 1.... d6. Wolfgang Zugrav asked whether this was a new book move but JONNY's operator answered that he had forgotten to set its parameter. Both players followed their own thoughts and played a remarkable opening. The irregularity of the position made it difficult to estimate the proper evaluation of the position. White won a pawn, but the black position seemed to be elastic, see Fig. 14c. 18. Rxf5 wins a pawn but Black still seems to have the better squares at its disposal. After rearranging the pieces, both players believed they had shown good play, see Fig. 14d.
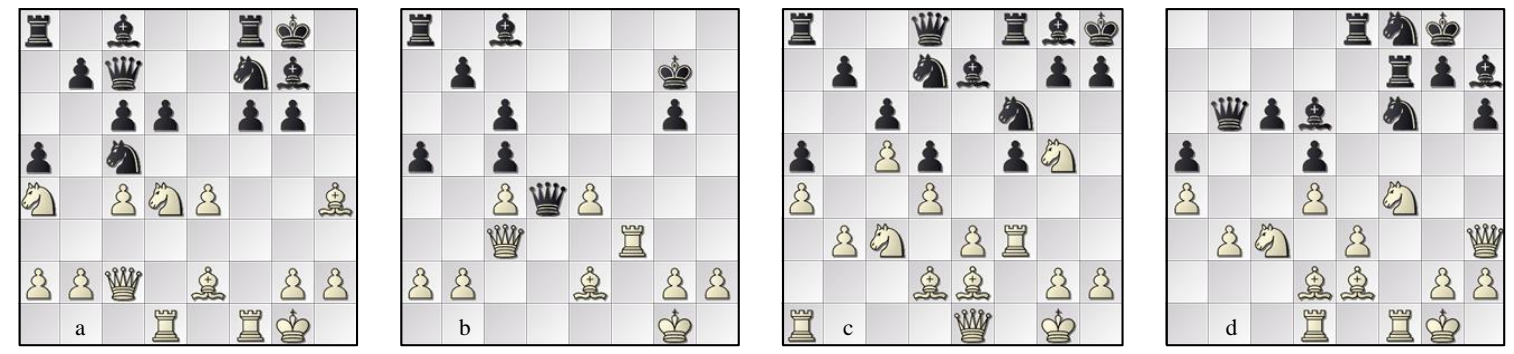

Fig. 14. Round 10: (a) THE BARON - KOMODO 18w and (b) 25w; (c) GINKGO - JONNY 18w and (d) 27 b.

Here Black decided to exchange the bishop for the knight by playing 27. ... Bxf4. White replied with 28. Rxf4 Ne6 29. Rff1 Bg6 30 Qh4 Qxb3. It is unclear why Black delayed taking on b3 for so long. Whatever the case, after this capture the material balance had been restored. Meanwhile the black position was to be preferred due to the weak e-pawn. Still, it took some time to regroup its pieces to start a final attack. Black was convinced of the power of this attack and therefore did not care to lose the exchange, see Fig. 15a. 
Black played 42. ... h3 which was followed by 43.g3 Nd6 44. Rb3 Nc4 45. Qa2 Qe7 and now Black had to take the exchange otherwise the rook moves to a free square. Meanwhile Black's position was so much improved that with easy combination it regained the exchange and kept the better position. Finally, on move 53, the weak e-pawn was captured and the game was considered lost by White. GINKGO resigned and so failed to walk through the door left open by KOMODO. Further, JONNY took over second place, another twist in the tale of the last round.
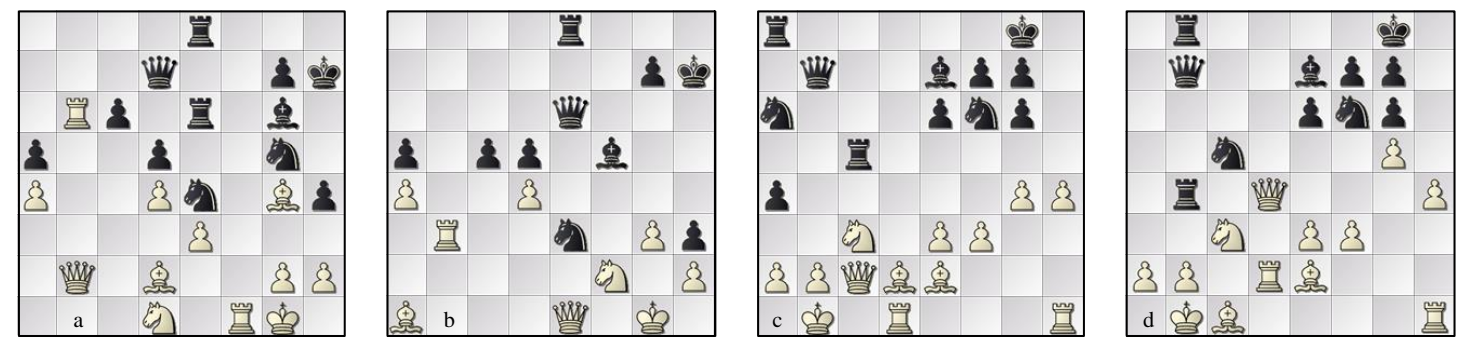

Fig. 15. Round 10: (a) GiNKGO - JoNNY 42b and (b) 54w; (c) CHIRON - SHREDDER 21w and (d) 26w.

The game CHIRON - SHREDDER followed a surprising path for spectators and computers. In the opening, the white player was quite assertive by taking the black a-pawn, opening lines to his king which had castled to the queenside as in Fig. 15c.

White played 21. Qxa4. The continuation was 21. ... Rc6 22. g5 Nc5 23. Qd4 Rb6 24. Bc1 Rb8 25. Rd2 Rb4. These moves led to the position of Fig. 15d.

There is heavy pressure on the b2 pawn and the queen is captured in the middle of the board. What could White expect from this position? White continued by taking the knight on $\mathrm{f} 6$ and retaking the rook on d4. Now two black pieces were hanging so the captured queen cost Black a rook and two pieces - of which one was regained. Here, Andrea (CHIRON) notes the RBP vs Q material imbalance and thinks SHREDDER undervalued White's three passed pawns. These would indeed have led to the reincarnation of the queen and a win but Black resigned first.

Final standings after R10: 1 KOMODO 61/2; 2= CHIRON, JONNY 6; 4 GINKGO 51/2; 5 SHREDDER 41/2; 6 THE BARON 11/2.

Table 2

The WCSC 2019 cross-table

\begin{tabular}{|c|c|c|c|c|c|c|c|c|c|c|c|c|}
\hline \# & id & Program & $\mathbf{K}$ & $\mathbf{J}$ & $\mathrm{C}$ & $\mathbf{G}$ & $\mathbf{S}$ & $\mathbf{T}$ & W & D & $\mathbf{L}$ & Score \\
\hline 1 & $\mathrm{~K}$ & KOMODO & & $1 / 21 / 2$ & $1 \frac{1}{2}$ & $1 / 21 / 2$ & $1 \frac{1 / 2}{2}$ & $11 / 2$ & 3 & 7 & 0 & $61 / 2$ \\
\hline 2 & $\mathrm{~J}$ & JONNY & $1 / 21 / 2$ & & $1 / 21 / 2$ & $1 / 21$ & $1 / 21 / 2$ & $1 \frac{1}{2}$ & 2 & 8 & 0 & 6 \\
\hline 3 & $\mathrm{C}$ & CHIRON & $01 / 2$ & $1 / 2^{1 / 2}$ & & $1 / 2^{1 / 2}$ & $1 / 21$ & 11 & 3 & 6 & 1 & 6 \\
\hline 4 & G & GINKGO & $1 / 22^{1 / 2}$ & $1 / 20$ & $1 / 21 / 2$ & & $1 / 21 / 2$ & 11 & 2 & 7 & 1 & $51 / 2$ \\
\hline 5 & S & SHREDDER & $01 / 2$ & $1 / 21 / 2$ & $1 / 20$ & $1 / 21 / 2$ & & $1 / 21$ & 1 & 7 & 2 & $41 / 2$ \\
\hline 6 & $\mathrm{~T}$ & THE BARON & $0^{1 / 2}$ & $01 / 2$ & 00 & 00 & $1 / 20$ & & 0 & 3 & 7 & $1 \frac{1}{2}$ \\
\hline
\end{tabular}

Congratulations go to KoMODO, the 2019 World Chess Software Champion, to JONNY and CHIRON, also on the podium - and to all the participants for creating an absorbing championship. The pgn file of the games, almost all closely contested and some with exemplar continuations, are available with the persistent e-repository version of this report (Krabbenbos et al., 2019b). 
Table 3

The WCSC 2019 results, round by round

\begin{tabular}{|c|c|c|c|c|c|c|c|c|c|c|c|c|}
\hline$\#$ & id & Program & r1 & r2 & r3 & r4 & r5 & r6 & r7 & r8 & r9 & r10 \\
\hline 1 & K & KOMODO & Sw 1 & $\mathrm{Jb}^{1 / 2}$ & $\mathrm{Gw}^{1 / 2}$ & $\mathrm{Cb} 1$ & Tw1 & $\mathrm{Sb}^{1 / 2}$ & $\mathrm{JW}^{1 / 2}$ & $\mathrm{~Gb}^{1 / 2}$ & $\mathrm{CW}^{1 / 2}$ & $\mathrm{~Tb}^{1 / 2}$ \\
\hline 2 & $\mathrm{~J}$ & JONNY & $\mathrm{Cb}^{1 / 2}$ & $\mathrm{Kw}^{1 / 2}$ & $\mathrm{Sb}^{1 / 2}$ & Tb1 & $\mathrm{Gw}^{1 / 2}$ & $\mathrm{Cw}^{1 / 2}$ & $\mathrm{~Kb}^{1 / 2}$ & $\mathrm{Sw}^{1 / 2}$ & $\mathrm{Tw}^{1} 1 / 2$ & Gb1 \\
\hline 3 & $\mathrm{C}$ & CHIRON & $\mathrm{JW}_{\mathrm{W}}^{1 / 2}$ & $\mathrm{~Gb}^{1 / 2}$ & Tw1 & Kw0 & $\mathrm{Sb}^{1 / 2}$ & $\mathrm{Jb}^{1 / 2}$ & $\mathrm{Gw}^{1} / 2$ & Tb1 & $\mathrm{Kb}^{1 / 2}$ & Sw1 \\
\hline 4 & G & GINKGO & Tw1 & $\mathrm{Cw}^{1 / 2}$ & $\mathrm{~Kb}^{1 / 2}$ & $\mathrm{Sw}^{1 / 2}$ & $\mathrm{Jb}^{1 / 2}$ & Tb1 & $\mathrm{Cb}^{1} / 2$ & $\mathrm{Kw}^{1 / 2}$ & $\mathrm{Sb}^{1} / 2$ & Jw0 \\
\hline 5 & S & SHREDDER & $\mathrm{Kb} 0$ & $\mathrm{~Tb}^{1 / 2}$ & $\mathrm{JW}^{1 / 2}$ & $\mathrm{~Gb}^{1 / 2}$ & $\mathrm{Cw}^{1 / 2}$ & $\mathrm{Kw}^{1 / 2}$ & Tw1 & $\mathrm{Jb}^{1 / 2}$ & $\mathrm{Gw}^{1 / 2}$ & $\mathrm{Cb} 0$ \\
\hline 6 & $\mathrm{~T}$ & THE BARON & Gb0 & $\mathrm{Sw}^{1 / 2}$ & $\mathrm{Cb} 0$ & Jw0 & $\mathrm{Kb} 0$ & Gw0 & $\mathrm{Sb} 0$ & Cw0 & $\mathrm{Jb}^{1} 1 / 2$ & $\mathrm{Kw}^{1 / 2}$ \\
\hline
\end{tabular}

Table 4

WCSC 2019 progress, round by round

\begin{tabular}{cccccccccccc}
\hline$\#$ & Program & r1 & r2 & r3 & r4 & r5 & r6 & r7 & r8 & r9 & r10 \\
\hline 1 & KOMODO & 1 & $11 / 2$ & 2 & 3 & 4 & $41 / 2$ & 5 & $51 / 2$ & 6 & $61 / 2$ \\
2 & JONNY & $1 / 2$ & 1 & $1 \frac{1}{2} 2$ & $2 \frac{1}{2} 2$ & 3 & $31 / 2$ & 4 & $41 / 2$ & 5 & 6 \\
3 & CHIRON & $1 / 2$ & 1 & 2 & 2 & $21 / 2$ & 3 & $31 / 2$ & $41 / 2$ & 5 & 6 \\
4 & GINKGO & 1 & $11 / 2$ & 2 & $21 / 2$ & 3 & 4 & $41 / 2$ & 5 & $51 / 2$ & $51 / 2$ \\
5 & SHREDDER & 0 & $1 / 2$ & 1 & $11 / 2$ & 2 & $21 / 2$ & $31 / 2$ & 4 & $41 / 2$ & $41 / 2$ \\
6 & THE BARON & 0 & $1 / 2$ & $1 / 2$ & $1 / 2$ & $1 / 2$ & $1 / 2$ & $1 / 2$ & $1 / 2$ & 1 & $1 \frac{1}{2} 2$ \\
\hline
\end{tabular}

\section{REFERENCES}

CPW (2019). https://tinyurl.com/icga046. Biographies of programs, authors and operators.

Krabbenbos, J., van den Herik, H.J. and Haworth, G.McC. (2019a). WCCC 2019: the $25^{\text {th }}$ World Computer Chess Championship. http://centaur.reading.ac.uk/85845/. ICGA Journal, 41(4), 206-221. doi: 10.3233/ICG-190125.

Krabbenbos, J., van den Herik, H.J. and Haworth, G.McC. (2019b). This note with an annotated pgn file, available from http://centaur.reading.ac.uk/85846/. ICGA Journal, 41(4), 222-236. doi: 10.3233/ICG-190126.

Krabbenbos, J., van den Herik, H.J. and Haworth, G.McC. (2019c). WSCC 2019: the 2019 World Speed Computer Chess Championship. http://centaur.reading.ac.u/85847/. ICGA Journal, 41(4), 237240. doi: 10.3233/ICG-190127. 